\title{
Correlação entre achados macro e microscópicos em 200 autópsias consecutivas: análise do valor custo/benefício do estudo histopatológico completo das autópsias
}

\author{
Relationship between gross and microscopic findings in 200 consecutive autopsies: cost/benefit value of the \\ histopathological study of all organs and systems
}

\author{
Leandro Santini Echenique \\ Regina de Arruda Mello ${ }^{2}$ \\ Luciana Nakao Odashiro ${ }^{2}$ \\ Marcello Franco ${ }^{3}$
}

\begin{tabular}{|c|c|}
\hline unitermos & \\
\hline $\begin{array}{l}\text { Autópsia } \\
\text { Histopatologia } \\
\text { Custo }\end{array}$ & $\begin{array}{l}\text { Introdução: Apesar dos avanços na área da medicina diagnóstica, a discrepância entre os } \\
\text { diagnósticos clínicos e os da autópsia tem permanecido em torno de } 10-20 \% \text {. É importante } \\
\text { reverter esta tendência por medidas que valorizam a realização e a otimização das autópsias. } \\
\text { Objetivos: Comparar prospectivamente os achados macro e microscópicos de } 200 \text { autópsias, } \\
\text { visando analisar o impacto e a relação custo/benefício do estudo histopatológico de todos os } \\
\text { órgãos sobre os diagnósticos provisórios macroscópicos e sobre os laudos finais de encerra- } \\
\text { mento. Metodologia: Analisamos } 200 \text { autópsias consecutivas realizadas no Departamento de } \\
\text { Patologia da Escola Paulista de Medicina/Unifesp, e avaliamos a concordância e a } \\
\text { discordância entre os diagnósticos macroscópicos provisórios e os microscópicos finais. } \\
\text { Resultados: Houve concordância entre os diagnósticos macro e microscópicos em } 143 \text { casos } \\
\text { (71,5\%) e discordâncias em } 22 \text { casos ( } 11 \% \text { ), classificadas como leves em } 14 \text { casos (7\%) e } \\
\text { graves em oito casos (4\%). Em } 35 \text { casos (17,5\%), a histopatologia revelou alterações sem } \\
\text { expressão macroscópica e cujo diagnóstico final foi histológico. Conclusão: O elevado índice } \\
\text { de concordância detectado entre os diagnósticos macro e microscópicos, sendo a maioria } \\
\text { das discordâncias classificada como leve, parece indicar que as autópsias podem ser encerra- } \\
\text { das com estudo histopatológico limitado às alterações macroscópicas mais evidentes, com } \\
\text { significativa redução de custo (cerca de } \$ \text { \$ } 300,00 \text { por autópsia) e grande melhoria no } \\
\text { retorno, em curto período de tempo, da informação para o corpo clínico da instituição. }\end{array}$ \\
\hline
\end{tabular}

abstract

Background: Despite the development in diagnostic medicine, discrepancy between clinical diagnoses and the ones obtained by autopsy has remained around 10-20\%. It is important to revert this tendency by measures that value the realization and optimization of autopsies. Objectives: Prospectively compare gross and microscopic findings of 200 autopsies, viewing to analyze the impact and the cost/benefit value of the histopathological study of all organs over provisory microscopic diagnoses and over enclosure final awards. Methods: We analyzed 200 consecutive autopsies performed at Departamento de Patologia da Escola Paulista de Medicina/ Unifesp and evaluated agreement and disagreement between provisory gross diagnoses and final microscopic ones. Results: There was agreement between gross and microscopic findings in 143

cases (71.5\%) and disagreement in 22 cases (11\%), classified as light in 14 cases (7\%) and serious in eight cases (4\%). In 35 cases (17.5\%), histopathological study revealed alterations with no gross significance which had final histological diagnosis. Conclusion: The high agreement index

detected between gross and microscopic findings, most discrepancies being classified as light, seems to indicate that autopsies may be closed with histopathological study limited to the most evident gross alterations, with significant cost reduction (around $R \$ 300$ per autopsy) and great improvement in the return, in a short period of time, of information to the institution clinical staff. 


\section{Introdução}

O declínio no número de autópsias, em hospitais e instituições universitárias, é evento amplamente reportado, inicialmente em países do primeiro mundo $(2,7,9)$ e posteriormente em muitos países em desenvolvimento, como o Brasil (11). As causas para esta tendência são múltiplas e complexas, incluindo aspectos religiosos, familiares e médicos (8). Entre estes últimos, salientam-se razões diagnósticas, caracterizadas pelo grande avanço tecnológico na imagenologia $(22,26,27)$, com a suposição de que todos os diagnósticos agora podem ser feitos em vida, e pela crescente ansiedade de processos judiciais contra os médicos por má prática $(3,13,15,20)$.

Entretanto, apesar dos avanços nas técnicas e na medicina diagnóstica, o nível de discrepância entre os diagnósticos clínicos e os de autópsia $(5,25)$ tem permanecido em torno de $10 \%$ a $20 \%$. Este fato é ainda mais acentuado entre a população idosa, onde a porcentagem de condições que não são diagnosticadas e que seriam potencialmente tratadas é ainda maior $(6,10,12,14,18$, $21,28)$.

O impacto destes níveis de discrepância nas estatísticas de mortalidade, frente ao acentuado declínio no número de autópsias, é preocupante $(4,19)$. Neste sentido, muitas investigações têm demonstrado que os diagnósticos baseados nas autópsias são necessários para garantir a acurácia dos atestados de óbito e, portanto, das estatísticas de mortalidade geradas a partir destes documentos $(1,16,17)$

As autópsias são procedimentos caros; envolvem dependências e equipamentos especiais, corpo médico e técnico especializado, muitas horas de trabalho na realização, preparo das peças, seguimento dos fragmentos para histopatologia, exame das lâminas, feitura dos relatórios macro e microscópicos, apresentação e discussão dos achados em reuniões anatomoclínicas, etc. (24).

O custo de todos estes procedimentos tem sido também apontado como uma das razões para o desinteresse dos órgãos médicos na montagem e manutenção de serviços de autópsia (10).

No Hospital São Paulo, o número de autópsias de adultos tem declinado nos últimos anos, com média anual de 321 , no período de 1990-1994, para média anual de 234, no período de 1995-2000. Temos tentado reverter esta tendência através de medidas que valorizam a realização de autópsias, como: 1) reuniões semanais para a discussão dos achados macroscópicos, com a elaboração de lau- dos macroscópicos provisórios, que são imediatamente encaminhados aos médicos solicitantes; 1) reuniões semanais de apresentação dos achados microscópicos das autópsias checadas macroscopicamente na semana anterior, visando acelerar o encerramento e a elaboração dos relatórios finais; 3) reuniões anatomoclínicas com os diferentes serviços do hospital, para a transferência ao corpo clínico de todas as informações e achados pertinentes, e também como mecanismo de controle de qualidade das interpretações morfológicas sugeridas $(23,28,29)$.

\section{Objetivo}

Uma observação que surgiu da rotina de autópsias no nosso serviço foi que o custo poderia ser significativamente reduzido se otimizássemos o estudo histopatológico de todas as autópsias. Ao contrário da rotina antiga e clássica de seguir fragmentos de todos os órgãos, independente de eles exibirem alterações morfológicas, procedimento que acaba por representar a inclusão de 30 a 40 amostras, com a respectiva sobrecarga de trabalho para o corpo técnico e para o patologista, resolvemos testar a hipótese de que a maioria das autópsias poderia ser encerrada microscopicamente, sem prejuízo da qualidade das mesmas, com um número menor e selecionado de fragmentos, escolhidos criteriosamente em função da presença ou não de alterações macroscópicas óbvias e do contexto da doença principal e das causas conseqüenciais.

Este é o objetivo principal do presente trabalho, onde comparamos prospectivamente os achados macro e microscópicos de autópsias de adultos, visando analisar o impacto do estudo histopatológico de todos os órgãos e sistemas sobre os diagnósticos provisórios macroscópicos (fase pré-microscopia) e sobre os laudos finais de encerramento das autópsias (fase pós-microscopia).

\section{Material e métodos}

Foram analisadas 200 autópsias consecutivas de adultos com idade superior a 18 anos, realizadas no Departamento de Patologia da Universidade Federal de São Paulo/Escola Paulista de Medicina, durante o período de 1998 e 1999.

O protocolo do exame dos achados macro e microscópicos das autópsias foi o seguinte: 1) o conjunto das peças macroscópicas das autópsias era guardado em formalina a 10\% para ser apresentado em reunião semanal de macroscopia, onde todos os casos da semana eram 
discutidos com os docentes e residentes do departamento. O objetivo era elaborar laudo provisório dos achados, com a definição da doença básica, causas conseqüenciais, causa de morte, causas contributivas e outras doenças; 2) ao final da reunião, as autópsias seguiam para exame microscópico, com a inclusão de fragmentos de todos os órgãos; 3) na semana seguinte, o resultado deste estudo microscópico era apresentado em reunião de microscopia das autópsias, com o objetivo de checar se todas as dúvidas haviam sido esclarecidas e de elaborar o laudo final, macro e microscópico, das autópsias, com a correlação anatomoclínica final.

Baseados nos laudos assim emitidos, realizamos estudo de correlação entre os diagnósticos macroscópicos provisórios e os diagnósticos microscópicos finais, procurando definir o nível de concordância e discordância entre eles, segundo os seguintes parâmetros:

- diagnóstico concordante: quando o estudo histopatológico confirmou os diagnósticos macroscópicos;

- diagnóstico discordante: quando o estudo histopatológico revelou alterações não-suspeitadas ou erroneamente diagnosticadas pelo exame macroscópico. Esta discordância foi subclassificada em leve, quando as possíveis repercussões do erro poderiam ser consideradas de menor importância no contexto geral da autópsia, ou em grave, quando o erro na interpretação macroscópica dos achados poderia implicar em significativo impacto na avaliação da morbidade e incidência das condições mórbidas, em termos de estatística de doenças em nosso meio.
Portanto, o objetivo principal desta abordagem foi definir o escopo máximo do estudo histopatológico para cada grupo de doenças, sem prejuízo para a qualidade final do processo.

\section{Resultados}

Das 200 autópsias analisadas, houve concordância entre os diagnósticos provisórios e finais das autópsias em 143 casos (71,5\%). Na maioria das autópsias concordantes, predominaram casos de neoplasia (30 casos; 21\%), ou do complexo hipertensão arterial, aterosclerose e diabetes melito (27 casos; 18,8\%). A seguir foram observados casos da síndrome da imunodeficiência adquirida (16 casos; $11,2 \%$ ) e de tuberculose (14 casos; 9,8\%) (Tabela 1).

Foram observadas discordâncias entre os diagnósticos macro e microscópicos em 22 casos (11\%). Estas discordâncias foram consideradas leves em 14 casos (7\%) e graves em oito casos (4\%) (Tabelas 2 e 3 ).

Todas as discordâncias leves envolveram afecções pulmonares, salientando-se a dificuldade no diagnóstico macroscópico de broncopneumonia.

A maioria dos casos com discordância grave revelou dificuldade no diagnóstico macroscópico de tuberculose, condição que não foi diagnosticada ou o foi erroneamente em seis casos (75\%).

Em 35 casos (17,5\%), o estudo histopatológico revelou alterações sem expressão macroscópica, ou cujo diagnóstico final foi essencialmente histológico (Tabela 4). A

\section{Relação das autópsias com diagnósticos macro e microscópicos concordantes em 200}

Tabela 1 autópsias consecutivas

\section{Diagnóstico macroscópico}

Neoplasias

Doenças hipertensiva-ateroscleróticas com ou sem diabetes melito

Síndrome da imunodeficiência adquirida

Tuberculose

Cardiopatias

Cirrose hepática

Lúpus eritematoso sistêmico

Sepse

Trombose venosa profunda

Outros

\section{Número de casos}

3

27

16

14

13

10

6

4

3

20

143

$\%$

21

18,8

11,2

9,8

9,1

7

4,2

2,8

2,1

14

Total 
Relação das autópsias com discordância leve entre os diagnósticos macro e microscópicos

Tabela 2 em 200 autópsias consecutivas

\begin{tabular}{llcc}
\hline \multicolumn{1}{c}{ Diagnóstico } & \multicolumn{1}{c}{ Total de casos } & $\%$ \\
\hline Macroscópico & Microscópico & & \\
\hline Pulmão normal ou edematoso & Broncopneumonia & 5 & 33,7 \\
Tromboembolismo pulmonar & Congestão pulmonar & 2 & 14,28 \\
Broncopneumonia & Hemorragia & 2 & 14,28 \\
SDRA* & Congestão e hemorragia pulmonar & 1 & 7,14 \\
Pneumonia lobar & Broncopneumonia & 1 & 7,14 \\
Pulmão normal & Edema agudo de pulmão & 1 & 7,14 \\
Broncopneumonia & SDRA & 1 & 7,14 \\
Hemorragia pulmonar & Êmbolos sépticos & 1 & 7,14 \\
\hline Total & & 14 & 100 \\
\hline
\end{tabular}

*Síndrome do desconforto respiratório agudo

\section{Relação das autópsias com discordância grave entre os achados macro e microscópicos}

Tabela 3 em 200 autópsias consecutivas

\begin{tabular}{llcl}
\hline & Diagnóstico & Total de casos & $\%$ \\
Macroscópico & Microscópico & \\
Pulmão normal & Tuberculose & 3 & 37,5 \\
Broncopneumonia & Tuberculose & 1 & 12,5 \\
Tuberculose pulmonar & Broncopneumonia & 1 & 12,5 \\
Tuberculose pulmonar & Adenocarcinoma de pulmão & 1 & 12,5 \\
Pneumonia lobar & Pneumonia intersticial & 1 & 12,5 \\
Glioma & Acidente vascular cerebral & 1 & 12,5 \\
\hline Total & & $\mathbf{8}$ & $\mathbf{1 0 0}$ \\
\hline
\end{tabular}

Tabela 4

Relação das autópsias onde 0 estudo microscópico revelou alteraçōes sem expressão

\begin{tabular}{llcc}
\hline \multicolumn{1}{c}{ Diagnóstico } & Total de casos & $\%$ \\
Macroscópico & Microscópico & & \\
Infecção & Definição do agente infeccioso & 18 & 51,4 \\
Pulmão normal & Microtrombos pulmonares & 6 & 17,1 \\
Ausência de envolvimento metastático & Metástases histológicas & 4 & 11,4 \\
Coração ou rim normal & Rejeição aguda ou crônica & 2 & 5,7 \\
Neoplasia disseminada sem sítio definido & Definição do sítio primário & 2 & 5,7 \\
Linfoma & Proliferação linfóide atípica reacional & 1 & 2,9 \\
Tromboembolismo & TEP por coriocarcinoma metastático & 1 & 2,9 \\
Carcinomatose peritoneal & Pseudomixoma & 1 & 2,9 \\
\hline Total & & 35 & 100 \\
\hline
\end{tabular}


maioria dos casos envolveu a identificação histológica do agente causador da lesão. A histopatologia foi também importante na identificação de tromboembolismo pulmonar periférico, sem evidência clínica ou macroscópica de trombose venosa profunda ou tromboembolismo em ramos principais da artéria pulmonar.

\section{Discussão}

Nosso serviço de autópsia está inserido em complexo hospitalar universitário de grande centro urbano, onde a população atendida apresenta essencialmente as doenças mais prevalentes no mundo ocidental, ou seja, aterosclerose e neoplasias.

As autópsias de pacientes falecidos em conseqüência de aterosclerose e de suas complicações, em associação eventual com hipertensão arterial e diabetes, apresentam alterações macroscópicas bem definidas, de fácil identificação, que, em geral, não requerem exame histológico para confirmação diagnóstica. Assim, número significativo de diagnósticos concordantes entre os achados macro e microscópicos foi detectado em autópsias cujo diagnóstico de doença básica incluiu condições do complexo hipertensão arterial, aterosclerose e diabetes.

O mesmo ocorreu em autópsias de neoplasias, particularmente carcinomas, onde o diagnóstico macroscópico presuntivo foi confirmado pela histopatologia. Muitos destes casos, também, já tinham exames laboratoriais préóbito que definiram o tipo de neoplasia, o que possibilitou a correta interpretação dos achados macroscópicos da autópsia. Neste grupo de pacientes, seria possível reduzir significativamente o material a ser seguido para estudo histológico, o que acarretaria menor custo da autópsia, com economia de material de consumo e de mão-de-obra. Desta maneira também otimizaríamos a finalização das autópsias, fato que estimularia clínicos e familiares a pedir e a autorizar mais freqüentemente tal procedimento (24).

Os casos discordantes foram divididos em discrepância leve ou grave, segundo o nível de impacto que o erro poderia causar nas estatísticas de morbidade.

As discrepâncias leves envolveram principalmente dificuldades na correta interpretação macroscópica de alterações pulmonares, onde há certo grau de subjetividade na avaliação diferencial entre condições de edema, congestão e hemorragia pulmonares, broncopneumonia e dano alveolar difuso. Este achado demonstra a importância do exame histopatológico do pulmão em situações onde o diagnóstico diferencial entre estas entidades é essencial.

A maioria das autópsias com discrepâncias graves entre os achados macro e microscópicos envolveu dificuldades no diagnóstico correto de tuberculose, que ou não foi reconhecida na reunião semanal de macroscopia, ou foi interpretada erroneamente, tendo sido confundida com outra afecção. Provavelmente este fato reflete a não-familiaridade do staff com esta condição infecciosa, que passou a ser raramente vista na mesa de autópsia, até que a epidemia de Aids e o aumento de outras causas de imunodepressão causaram recrudescência e aparecimento de formas atípicas da doença.

É importante ressaltar que o exame histopatológico foi, em $17,5 \%$ dos casos, essencial para a correta interpretação dos achados macroscópicos porque revelou alterações que não têm manifestação macroscópica, cujo diagnóstico é essencialmente histológico. Neste grupo estão incluídos principalmente os casos $(45,7 \%)$ onde tinha sido feito o diagnóstico de processo infeccioso, porém a etiologia só pôde ser definida pelo estudo histopatológico.

$\mathrm{O}$ presente trabalho visou adequar o procedimento rotineiro das necropsias à situação econômica da saúde pública, já que cada lâmina corada pela hematoxilina e pela eosina custa cerca de $\mathrm{R} \$ 10,00$ (informação pessoal, Associação Brasileira dos Laboratórios de Anatomia Patológica e Citopatologia - Abralapac) e uma necropsia com representação de todos os órgãos de maneira ideal confeccionaria cerca de 40 lâminas, como preconizado em Manual sobre Autópsias, da Sociedade Brasileira de Patologia. Já com o estudo racionalizado como aqui proposto, seriam confeccionadas cerca de dez lâminas, levando a uma economia de cerca de $\mathrm{R} \$ 300,00$ por autópsia.

O estudo visou também recolocar a autópsia como procedimento fundamental de controle de qualidade hospitalar. Tal importância só será reconhecida quando este método for visto como complementar e de aprendizado para o corpo clínico, e não como método punitivo. Uma das formas de alcançar estes resultados é fazer com que clínicos e familiares recebam o retorno do procedimento realizado no menor período de tempo e com as dúvidas, anteriormente existentes, sanadas através de relatórios finais concisos e objetivos.

Sabemos das vantagens de se realizar o estudo histopatológico completo das autópsias, com amostragem de todos os órgãos, inclusive daqueles sem alterações macroscópicas, particularmente nos hospitais universitários com residência médica em patologia. Infelizmente 
conhecemos também a difícil realidade econômica atual de nosso país, com a necessidade de diminuição dos custos da assistência médica em todos os setores, tanto em instituições públicas quanto privadas. Com a presente proposta, pretendemos contribuir para o resgate da importância das autópsias, que seriam realizadas com menor custo e em menor período de tempo, pois achamos preferível vê-las realizadas com recursos reduzidos à sua ausência completa.
Outra maneira de também alcançar tais resultados pressupõe progressos tecnológicos e apoio financeiro ainda maior, como exames de imagem, documentação fotográfica e congelação na sala de necropsia.

\section{Agradecimentos}

Os autores agradecem ao prof. Thales de Brito e ao dr. Venâncio A. Alves pelas sugestões ao manuscrito.

\section{Referências}

I. Almeida, M.C. et al. Correlação diagnóstica anatomoclínica: aferição retrospectiva do diagnóstico clínico em necropsias. Ver. Saúde Públ., 23: 285-91, 1989.

2. Avis, S.P. Death investigation in Canadá.J. Forensic Sci., 43: 377-9, 1998.

3. Black, W.C. \& Welch, G. Advances in diagnostic imaging and overestimations of disease prevalence and the benefits of therapy. N. Engl. J. Med., 328: 1237-43, 1993.

4. Blosser, S.A. et al. Do autopsies of critically ill patients reveal important findings that were clinically indetected? Crit. Care Med., 26: 1332-6, 1998.

5. Britton, M. Diagnostic errors discovered at autopsy. Acta Med. Scand., 196: 203-10, 1974.

6. Burton, E.C. et al. Autopsy diagnoses of malignant neoplasms: how often are clinical diagnoses incorrect? JAMA, 280: 1245 8, 1998.

7. Cheng, T.O. The autopsy: still important. Ann. Thorac. Surg., 65: 597-8, 1998.

8. Cottrill, H.M. \& O'Connor, W.N.The autopsy in the $21^{\text {st }}$ century: time for reconsideration.J. Ky Med. Assoc., 98: I I 0-4, 2000.

9. Cross, S.The autopsy in the 1990's. Br.J. Hosp. Med., 58: 598, 1998.

I 0. Friederici, H.H.R. \& Sebastian, M.Autopsies in a modern teaching hospital. Arch. Pathol. Lab. Med., 108: 518-21, 1984.

I I. Gambino, S.R. The autopsy. Arch. Pathol. Lab. Med., I08: 444-5, 1984.

12. Gobbato, F. et al. Inaccuracy of death certificate diagnoses in malignancy: an analysis of I 405 autopsied cases. Hum. Pathol., 13: 1036-8, 1982

13. Goldman, L. Diagnostic advances the value of the autopsy. Arch. Pathol. Lab. Med., I 08: 501-5, 1984.

14. Goldman, L. et al. The value of the autopsy in three medical eras. N. Engl. J. Med., 308: 1000-5, 1983.

15. Hasson, J. Medical fallibility and the autopsy in the USA. J. Eval. Clin. Pract., 3: 229-34, 1997.

16. Hasuo, Y. et al. Accuracy of diagnosis on death certificates for underlying causes of death in a long-term autopsy-based population study in Hisayama, Japan, with special reference to cardiovascular disease.J. Clin. Epidemiol., 42:577-84, 1989.

17. Kircher, T. et al. The autopsy as a measure of accuracy of the death certificate. N. Engl. J. Med., 3/ 3: I263-9, 1985.

18. Lynn, J. et al. Autopsy rates and diagnosis. JAMA, 281 (23): $2|8|-5,1999$
19. Lundberg, G.D. Medicine without the autopsy. Arch. Pathol. Lab. Med., 108: 449-54, 1984.

20. Nemetz, P.N. et al. Assessing the autopsy. Am. J. Pathol., 128 : 362-79, 1987.

21. Pastores, S.M. \& Halpern, N.A. Autopsies in the ICU: we still need them! Crit. Care Med., 27: 235-6, 1999.

22. Scholmerich, J. et al. Effect of diagnostic imaging on pre-mortem diagnostic reliability. Med. Klin., 92: 394-400, 1997.

23. Segoviano, P.F. et al.Autopsy as quality assurance in the intensive care unit. Crit. Care Med., 16:683-5, 1988.

24. Sinard J.H. \& Blood, D.J. Quality improvement on an academic autopsy service. Arch. Pathol. Lab. Med., 125: 237-45, 200 I.

25. Stevanovic, G. et al. Correlation of clinical diagnoses with autopsy findings: a retrospective study of 2145 consecutive autopsies. Hum. Pathol., I 7: I 225-30, 1986.

26. Sonderegger, I.K. et al. Diagnostic errors in three medical eras: a necropsy study. Lancet, 355: 2027-31, 2000.

27.Tsujimura,T. et al.Why couldn't an accurate diagnosis be made? An analysis of 1044 consecutive autopsy cases. Pathol. Int. 49: 408-10, 1999.

28. Zarbo, R.J.The autopsy as a performance measurement tooldiagnostic discrepancies and unresolved clinical questions: a College of American Pathologists Q-Probes study of 2479 autopsies from 248 institutions. Arch. Pathol. Lab. Med., 123: 191-8, 1999.

29. Zehr, K.J. et al. The autopsy: still important in cardiac surgery. Ann. Thorac. Surg., 64: 380-3, 1997.
Endereço para correspondência

Marcello Franco

Depto. de Patologia - EPM/Unifesp

Rua Botucatu 740 - Vila Clementino

CEP 04023-900 - São Paulo-SP

Fax: (11) 571-9295

e-mail: mfranco@patologia.epm.br 